\title{
Relevance of the clustered regularly interspaced short palindromic repeats of Enterococcus faecalis strains isolated from retreatment root canals on periapical lesions, resistance to irrigants and biofilms
}

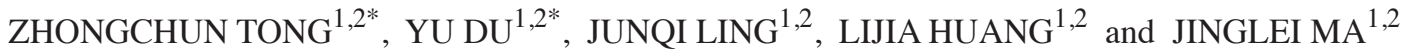 \\ ${ }^{1}$ Department of Operative Dentistry and Endodontics, Guanghua School of Stomatology; \\ ${ }^{2}$ Guangdong Provincial Key Laboratory of Stomatology, Sun Yat-sen University, Guangzhou, Guangdong 510055, P.R. China
}

Received September 3, 2015; Accepted October 18, 2017

DOI: $10.3892 /$ etm.2017.5205

\begin{abstract}
A high prevalence of Enterococcus faecalis (E.faecalis) is observed in teeth with root canal treatment failures. Clustered regularly interspaced short palindromic repeats (CRISPR) are widely distributed in prokaryotes that have adaptive immune systems against mobile elements, including pathogenic genes. The present study investigated the relevance of the CRISPR in E. faecalis strains isolated from retreated root canals on biofilms, periapical lesions and drug resistance. A total of 20 E. faecalis strains were extracted from the root canals of teeth referred for root canal retreatment. CRISPR-Cas loci were identified by two pairs of relevant primers and polymerase chain reaction. The susceptibility of the 20 isolated strains to intracanal irrigants was evaluated by 1- and 5-minute challenges with a mixture of a tetracycline isomer, an acid and a detergent (MTAD), 2\% chlorhexidine (CHX) and $5.25 \%$ sodium hypochlorite $(\mathrm{NaOCl})$. The microtiter plate assay and crystal violet staining were used to compare the biofilm formation of the E. faecalis isolate strains. Out of the 20 E. faecalis isolate strains, 5 strains that lacked CRISPR-cas determinants exhibited significant periapical lesions. Among the 15 strains containing CRISPR-cas determinants, 8 were isolated from root canals with inadequate fillings and 7 were isolated from root canals without any fillings. The five strains lacking CRISPR-cas loci were observed to be more resistant to
\end{abstract}

Correspondence to: Professor Junqi Ling, Department of Operative Dentistry and Endodontics, Guanghua School of Stomatology, Sun Yat-sen University, 56 Lingyuan West Road, Guangzhou, Guangdong 510055, P.R. China

E-mail: junqi_ling@hotmail.com

*Contributed equally

Key words: Enterococcus faecalis, clustered regularly interspaced short palindromic repeats, chlorhexidine, sodium hypochlorite, biofilm
MTAD and 2\% CHX than the 15 strains that had CRISPR-cas loci. All of the strains exhibited the same susceptibility to $5.25 \% \mathrm{NaOCl}$. Furthermore, the 5 strains lacking CRISPR-cas determinants generated more biofilm than the other 15 strains. Thus, the results of the present study suggested that $E$. faecalis root canal isolates lacking CRISPR-cas exhibit higher resistance to intracanal irrigants, stronger biofilm formation and generate significant periapical lesions.

\section{Introduction}

Post-treatment apical periodontitis is typically caused by diverse pathogens, including Gram-positive and Gram-negative bacteria, and is characterized by the appearance and persistence of apical periodontic lesions $(1,2)$. Microbiological culturing techniques and molecular biological methods have been employed to investigate the microbiota from post-treatment root canal infections and have demonstrated that Enterococcus faecalis (E. faecalis) was highly prevalent in teeth that had undergone unsuccessful root canal therapies $(3,4)$. E. faecalis possesses numerous virulence factors, including cytolysin, lytic enzymes, pheromones, aggregation substances and lipoteichoic acid, and transmits these virulence traits into other species, further contributing to apical periodontitis $(5,6)$. Furthermore, E. faecalis may overcome the challenges of starvation and intracanal medication and persist as a pathogenic bacterium in the root canal system (7). When an adequate nutrition supply becomes available, the starved E. faecalis recover, resulting in increased pathogenicity (8). Therefore, E. faecalis may serve an important role in contributing to endodontic treatment failure.

Bacteria-like eukaryotes usually have an adaptive immune system that facilitates sequence-specific immunity against exogenous mobile genetic elements. Clustered regularly interspaced short palindromic repeats (CRISPR) loci encode this system $(9,10)$. E. faecalis is a common nosocomial pathogen that generates pathogenicity and resistance to antibiotics by acquiring mobile genetic elements, including antibiotic resistance genes (11). It has been demonstrated that among strains of E. faecalis, CRISPR1-cas and CRISPR3-cas loci are variable, and an inverse correlation between the presence of 
CRISPR-cas loci and acquired antibiotic resistance has been observed in clinically isolated strains of E. faecalis $(11,12)$. CRISPR-cas supplies E. faecalis with a specific sequence and provides a defense against plasmids, phages, pathogenicity islands and other elements $(12,13)$. Multidrug-resistant enterococci usually lack complete CRISPR loci and maybe invaded by mobile genetic elements (13). The complete genome of Enterococcus faecalis V583, a vancomycin-resistant clinical isolate, indicated that up to $25 \%$ of its genome consists of mobile elements (14).

Burley and Sedgley (15) investigated CRISPR-cas determinants that existed in an endodontic and oral E. faecalis isolate and observed that the occurrence of CRISPR-cas determinants was significantly higher in endodontic and oral E. faecalis than in hospital-acquired strains. It has been determined that the absence of CRISPR-cas is associated with the presence of antibiotic resistance $(12,15)$. CRISPR-cas impedes mobile genetic elements, which may include pathogenic genes. As a result, the presence and absence of CRISPR-cas determinants might be associated with the pathogenicity of a E. faecalis root canal isolate strain, further contributing to periapical lesions $(16,17)$. The aim of the present study was to investigate the possible relevance of a CRISPR-cas determinant of the E. faecalis root canal isolate strain to post-treatment periapical lesions. In addition, the CRISPR-cas determinant associated with biofilm formation and the resistance of the E. faecalis root canal isolate strain were assessed.

\section{Materials and methods}

Strain isolation. A total of 20 E. faecalis strains were isolated from the root canals of 20 patients who visited the Department of Operative Dentistry and Endodontics, Guanghua School of Stomatology, Sun Yat-sen University (Guangzhou, China) from January 2013 to January 2015, and these patients underwent endodontic treatment for at least one year. The teeth had a complete coronal filling according to a previous study (18). The appearance of an apical zone in these 20 teeth was revealed by radiographs and periapical lesion zones were measured using Image-Pro Plus 6.0 (Media Cybernetics, Inc., Rockville, MD, USA). E. faecalis strains were sampled from the root canals as follows: Following rubber dam isolation, the operative field was disinfected with $2.5 \% \mathrm{NaOCl}$. Samples were harvested using sterile paper points and then transferred into 2xYT medium (Qingdao-Hope Bio-Technology Co., Ltd., Qingdao, China). E. faecalis were isolated by inoculation in KF streptococcal agar medium (Qingdao-Hope Bio-Technology Co., Ltd.) and streaking on Bile Esculin Azide agar (BEA; cat. no. 212205; BD Biosciences, Franklin Lakes, NJ, USA). Isolated strains were identified by their bile resistance, colony morphology, Gram staining characteristics and oxygen tolerance, as well as by 16 s ribosomal DNA according topolymerase chain reaction (PCR) analysis. Among the 20 E. faecalis isolates, 12 were from teeth with imperfect root canal fillings and 8 were from teeth without a previous root canal filling. The present study was approved by the Ethics Review Committee of Institute of Stomatological Research, Sun Yat-Sen University (Guangzhou, China). All patients signed a consent form to take part in the study.
CRISPR loci identification. CRISPR1-cas and CRISPR3-cas are two common CRISPR loci of E. faecalis $(12,15)$. To examine CRISPR loci, two pairs of relevant primers (Table I) were devised to detect CRISPR1 or CRISPR3 loci according to a procedure by Burley and Sedgley (15). Total DNA from the 20 E. faecalis isolates was extracted using an E.Z.N.A. ${ }^{\circledR}$ Bacterial DNA Kit (D3350; Omega Bio-Tek, Inc., Norcross, GA, USA). PCR amplification was performed using $50 \mu \mathrm{l}$ of each sample and Taq DNA Polymerase (ET101; Tiangen Biotech Co., Ltd., Beijing, China) according to a procedure by Burley and Sedgley (15). PCR products $(20 \mu \mathrm{l})$ were analyzed using agarose gel electrophoresis, purified with an Agarose Gel DNA Extraction kit (product no. 11696505001; Roche, Basel, Switzerland) and submitted for sequencing at BGI-Tech Solutions, Co., Ltd. (Shenzhen, China). The nucleotide sequences were exported by Chromas software (v2.6.1; Technelysium Pty Ltd., Brisbane, Queensland, Australia) and the online ClustalW2 multiple sequence alignment tool (www. ebi.ac.uk/Tools/msa/clustalw2) was used to perform nucleotide sequence alignments according to the National Center for Biotechnology Information GenBank database (www.ncbi. nlm.nih.gov).

Assay of antibacterial activity. A microplate dilution method was used to examine the susceptibility of the $20 \mathrm{E}$. faecalis isolates to three routine intracanal irrigants: $5.25 \% \mathrm{NaOCl}$, $2 \%$ chlorhexidine $(\mathrm{CHX})$ and mixture of tetracycline isomer acid and detergent (MTAD) consisting of 3\% doxycycline, $4.25 \%$ citric acid and $0.5 \%$ polysorbate 80 detergent. E. faecalis OG1RF was used as a control due to its intrinsic CRISPR loci. E. faecalis isolates and OG1RF were grown to the exponential phase at a bacterial concentration of $\sim 10^{9}$ colony-forming units $/ \mathrm{ml}$. Aliquots $(1 \mathrm{ml})$ of $E$. faecalis were centrifuged at $7,610 \mathrm{x}$ g at $4^{\circ} \mathrm{C}$ for $5 \mathrm{~min}$. The supernatant was discarded and pellets were subsequently washed twice with phosphate-buffered saline (PBS) and challenged by resuspension in $5.25 \% \mathrm{NaOCl}, 2 \% \mathrm{CHX}$ or MTAD for 1 or $5 \mathrm{~min}$. The survival rates were examined by a 10 -fold gradient dilution and plate counts. The antibacterial assay of each strain was repeatedly performed three times on different days.

Evaluation of biofilm formation. A microtiter plate assay and crystal violet staining were used to evaluate and compare the biofilm formation of E. faecalis isolates. The bacteria were grown in brain heart infusion broth, to the exponential phase and were diluted 1:200 in tryptic soy broth (TSB; BD Biosciences) with $1 \%$ glucose. Subsequently, $200 \mu$ l diluted bacterial culture was added to 96 -well microtiter plates and incubated at $37^{\circ} \mathrm{C}$ for $24 \mathrm{~h}$ to allow for biofilm formation. E. faecalis biofilms on the bottom of the wells were evaluated by crystal violet staining following the protocol described in a previous study (19). In brief, following washing with PBS, the biofilms on the bottom of the wells were stained with $0.1 \%$ crystal violet for $15 \mathrm{~min}$ and subsequently washed with sterile distilled water. The bound dye was released by addition of $33 \%$ acetic acid and measured at $570 \mathrm{~nm}$ using a microplate reader. Each test was repeated four times in three independent experiments.

Statistical analyses. SPSS v18.0 (SPSS, Inc., Chicago, IL, USA) was used to perform statistical analyses. In the biofilm 
Table I. CRISPR-Cas primers used for determining the CRISPR-cas determinant in Enterococcus faecalis root canal isolate strains.

\begin{tabular}{lllc}
\hline CRISPR loci & \multicolumn{1}{c}{ Primer sequence (forward) } & \multicolumn{1}{c}{ Primer sequence(reverse) } & Amplicon length (bp) \\
\hline CRISPR1-cas & ATGGGCTGGCGAACGGTAGTGGTT & TCATATCCCAAACTCTGGAACTCCT & 867 \\
CRISPR1-cas & GCGATGTTAGCTGATACAAC & CGAATATGCCTGTGGTGAAA & 315 \\
CRISPR3-cas & GCTGAATCTGTGAAGTTACTC & CTGTTTTGTCACCGTTGGAT & 258 \\
CRISPR3-cas & GATCACTAGGTTCAGTTATTT & CATCGATTCATTATTCCTCCAA & 224 \\
\hline
\end{tabular}

CRISPR, clustered regularly interspaced short palindromic repeats.

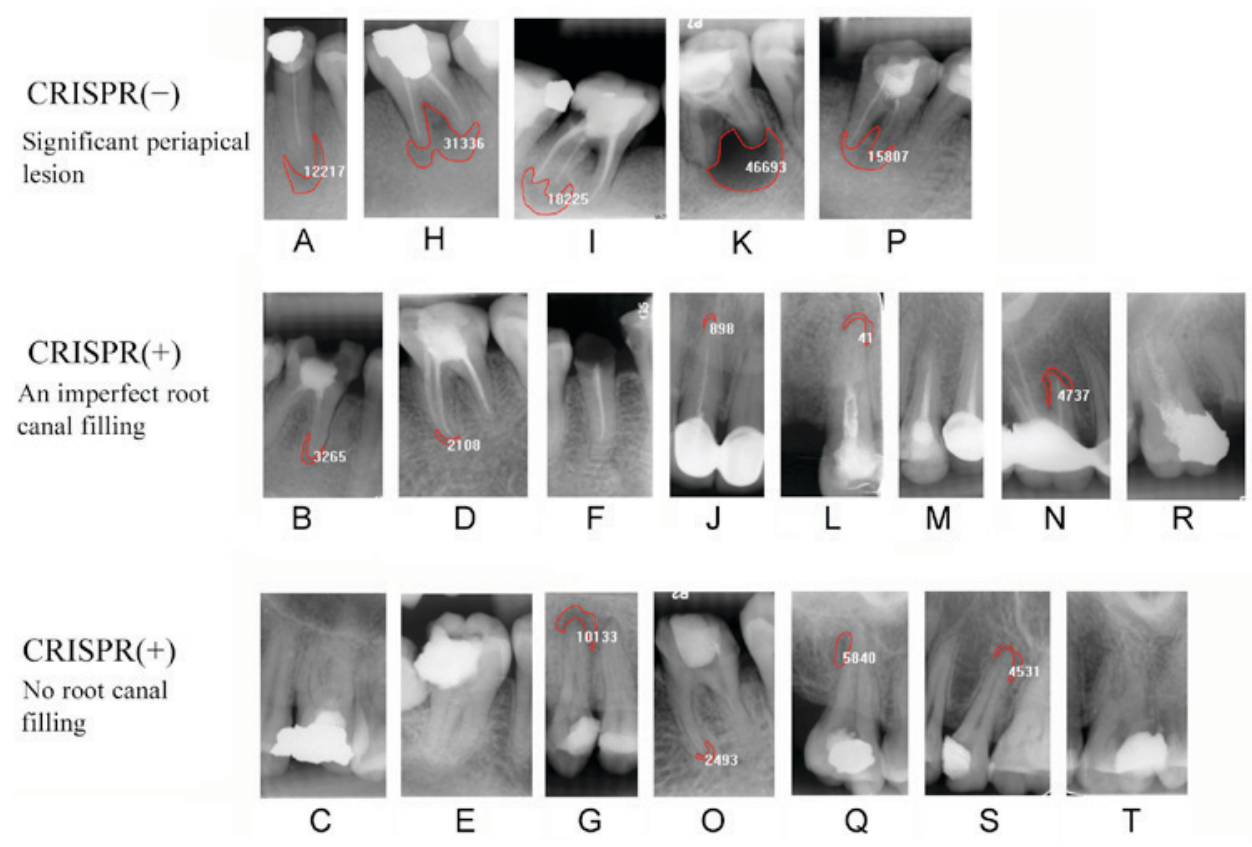

Figure 1. X-ray of periapical lesions in 20 teeth referred for root canal retreatment. The A, H, I, K, and P strains without a CRISPR-cas determinant were from teeth with significant periapical lesions, the B, D, F, J, L, M, N, and R strains in the presence of a CRISPR-cas determinant were from root canals with an inadequate filling, and the C,E, G, O, Q, S, and T strains with the presence of CRISPR-cas determinants were from root canals without a filling. CRISPR, clustered regularly interspaced short palindromic repeats.

assay, one-way analysis of variance and Tukey's honestly significant difference test were employed to compare the optical density (OD) values at $570 \mathrm{~nm}$ of the biofilm formation of 20 E. faecalis isolates and OG1RF. All values were expressed as the mean \pm standard deviation. $\mathrm{P}<0.05$ was considered to indicate a statistically significant difference.

\section{Results}

$X$-rays of 20 teeth with endodontic treatment. Out of the 20 E. faecalis isolate strains, the A, H, I, K, and P strains did not contain a CRISPR-cas determinant. X-rays of the corresponding teeth revealed significant periapical lesions and the area value of periapical lesions measured using Image-Pro Plus software was $>10,000$ IOD (integrated optical density). However, the other $15 \mathrm{E}$. faecalis isolate strains did contain CRISPR-cas determinants. Among these 15 strains, 8 strains were isolated from teeth with an imperfect root canal filling and 7 strains were isolated from teeth without a root canal filling. A majority of the 15 teeth exhibited a slight or moderate

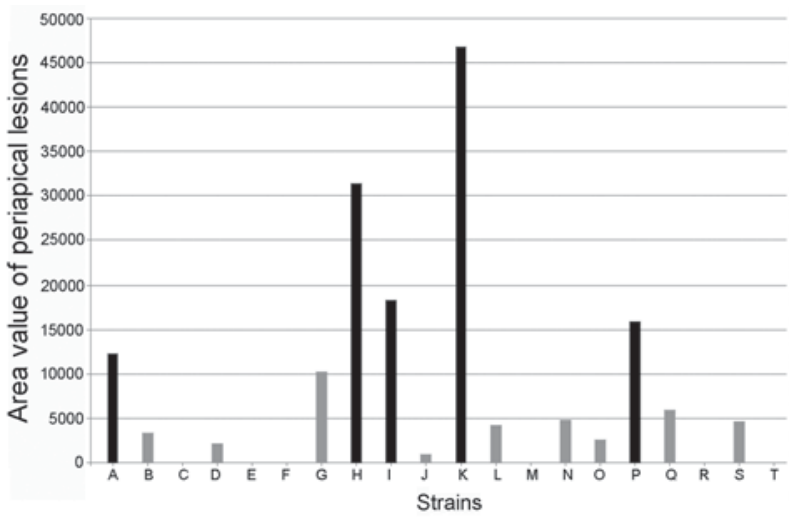

Figure 2. Periapical lesion area of 20 teeth referred for root canal retreatment. The appearance of an apical periodontitis lesion in the 20 teeth was revealed by radiographs and periapical lesion areas were measured using Image-Pro Plus software. The A, H, I, K, and P isolate strains lacking a CRISPR-cas determinant were from teeth with larger periapical lesion areas (black columns), while the other 15 isolate strains with the presence of a CRISPR-cas determinant were from teeth with smaller periapical lesions areas (gray columns). CRISPR, clustered regularly interspaced short palindromic repeats. 
Table II. Survival rates (\%) of 20 E. faecalis root canal isolate strains with or without CRISPR-cas determinants following 1- and 5-min challenges with routine intracanal irrigants.

\begin{tabular}{|c|c|c|c|c|c|c|c|}
\hline \multirow[b]{2}{*}{ E. faecalis } & \multirow[b]{2}{*}{ CRISPR } & \multicolumn{2}{|c|}{ MTAD } & \multicolumn{2}{|c|}{$2 \% \mathrm{CHX}$} & \multicolumn{2}{|c|}{$5.25 \% \mathrm{NaOCl}$} \\
\hline & & $1 \mathrm{~min}$ & $5 \mathrm{~min}$ & $1 \mathrm{~min}$ & $5 \mathrm{~min}$ & $1 \mathrm{~min}$ & $5 \mathrm{~min}$ \\
\hline A & $(-)$ & 7.20 & 1.00 & 5.20 & 0.32 & 0.012 & 0 \\
\hline B & $(+)$ & 2.50 & 0.12 & 0.80 & 0.05 & 0.0023 & 0 \\
\hline $\mathrm{C}$ & $(+)$ & 2.80 & 0.11 & 0.42 & 0.071 & 0.0037 & 0 \\
\hline $\mathrm{D}$ & $(+)$ & 0.50 & 0.11 & 0.24 & 0.04 & 0 & 0 \\
\hline $\mathrm{E}$ & $(+)$ & 1.20 & 0.21 & 2.60 & 0.12 & 0.0028 & 0 \\
\hline $\mathrm{F}$ & $(+)$ & 1.20 & 0.15 & 0.86 & 0.04 & 0.0085 & 0 \\
\hline G & $(+)$ & 1.80 & 0.09 & 0.58 & 0.02 & 0.0025 & 0 \\
\hline $\mathrm{H}$ & $(-)$ & 5.2 & 0.5 & 2.50 & 0.48 & 0.0008 & 0 \\
\hline I & $(-)$ & 8.0 & 1.1 & 4.20 & 0.36 & 0.0019 & 0 \\
\hline $\mathrm{J}$ & $(+)$ & 0.68 & 0.09 & 0.05 & 0.001 & 0.0013 & 0 \\
\hline $\mathrm{K}$ & $(-)$ & 6.10 & 0.98 & 4.20 & 0.80 & 0.0037 & 0 \\
\hline $\mathrm{L}$ & $(+)$ & 4.60 & 0.082 & 0.35 & 0.024 & 0 & 0 \\
\hline M & $(+)$ & 0.91 & 0.25 & 0.40 & 0.08 & 0.0005 & 0 \\
\hline $\mathrm{N}$ & $(+)$ & 3.50 & 0.34 & 1.50 & 0.034 & 0.0037 & 0 \\
\hline $\mathrm{O}$ & $(+)$ & 0.52 & 0.25 & 0.04 & 0.005 & 0.0025 & 0 \\
\hline $\mathrm{P}$ & $(-)$ & 2.90 & 1.15 & 4.80 & 0.52 & 0.0038 & 0 \\
\hline Q & $(+)$ & 0.084 & 0.008 & 2.40 & 0.057 & 0 & 0 \\
\hline $\mathrm{R}$ & $(+)$ & 1.50 & 0.16 & 1.20 & 0.15 & 0.0022 & 0 \\
\hline $\mathrm{S}$ & $(+)$ & 1.80 & 0.20 & 0.58 & 0.06 & 0.0064 & 0 \\
\hline $\mathrm{T}$ & $(+)$ & 0.95 & 0.04 & 0.38 & 0.10 & 0.0019 & 0 \\
\hline OG1RF & $(+)$ & 1.50 & 0.24 & 1.20 & 0.15 & 0.0028 & 0 \\
\hline
\end{tabular}

CHX, chlorhexidine; MTAD, mixture of $3 \%$ doxycycline, $4.25 \%$ citric acid and $0.5 \%$ polysorbate 80 detergent; E. faecalis, Enterococcus faecalis; CRISPR, clustered regularly interspaced short palindromic repeats.

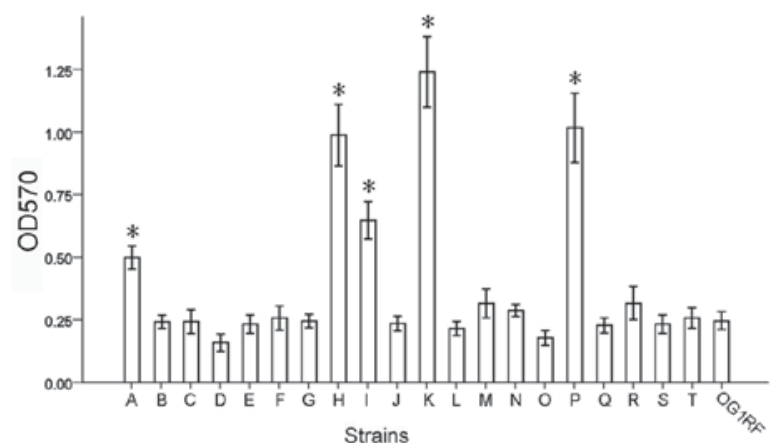

Figure 3. Biomass assay of biofilms in 20 different E. faecalis root canal isolate strains by crystal violet staining. The strain OG1RF was referred to as the control. ${ }^{*} \mathrm{P}<0.05$, biofilm biomass of the E. faecalis isolate strains vs. the control OG1RF. OD570, optical density at $570 \mathrm{~nm}$.

periapical lesion with an area of $<10,000$ IOD, with the exception of strain $\mathrm{G}$ (Figs. 1 and 2).

Susceptibility to antibacterial irrigants. Overall, the assay of antibacterial activity indicated that the 5 strains that did not contain CRISPR-cas loci were more resistant to MTAD or $2 \%$ CHX than the 15 strains that had CRISPR-cas loci; neither group exhibited significant differences in the $5.25 \% \mathrm{NaOCl}$ susceptibility assay. However, a among the 15 strains with CRISPR-cas loci, afew exceptional cases were encountered; for instance, the survival rates of strains $L$ and $N$ were 4.60 and $3.50 \%$ following a $1-$ min challenge with MTAD. The survival rates of strains $\mathrm{E}$ and $\mathrm{Q}$ were 2.60 and $2.40 \%$, respectively, following a 1-min challenge with $2 \% \mathrm{CHX}$ (Table II).

Biofilm formation ability. In the biofilm assay, the five strains (A, H, I, K and P) without CRISPR-cas loci had significantly higher OD values compared with the control OG1RF strain $(\mathrm{P}<0.05$; Fig. 3). Furthermore, the OD values of these strains were markedly higher compared with that of the other 15 isolate strains with CRISPR-cas determinants (Fig. 3).

\section{Discussion}

The CRISPR-cas module is a defense system against invading mobile genetic elements and is present in $\sim 40 \%$ of bacteria and $\sim 90 \%$ of archaea (20). The present study determined that among 20 test E. faecalis root canal isolates, 15 isolates presented with CRISPR-cas determinants, which was in accordance with results published by Burley and Sedgley (15) who demonstrated that 25 out of 34 endodontic isolates presented with CRISPR-cas determinants; however, these E. faecalis isolates were from teeth referred for primary, orthograde and 
endodontic treatment. In the present study, the CRISPR-cas determinant of $E$. faecalis isolates from teeth that were referred for root canal retreatment was investigated and it was observed that teeth infested with E. faecalis without CRISPR-cas determinants exhibited significant periapical lesions. The absence of CRISPR-cas determinants was associated with bacteriocin activity and a clumping response to pheromones in E.faecalis endodontic isolates (15). A clumping response to pheromones induces the generation of an aggregation substance and may lead to conjugative horizontal gene transfer, which causes transferable high-level antibiotic resistance and exposure to virulence determinants that are carried on plasmids $(21,22)$. Enterococci have been frequently identified in root canals with persistent periapical lesions and in teeth with insufficient root fillings $(23,24)$. Although enterococci have general ybeen observed in filled root canals without radiographic lesions, they are able to cause periapical lesions (25). The present study suggested that there may be a correlation between the CRISPR-cas determinant of E. faecalis and the degree of periapical lesions, which, however, may be caused by a number of factors.

CRISPR-cas systems mediate immunity to invading exogenous mobile genetic elements in three distinct stages: Adaptation, expression and interference (20). CRISPR-cas systems capture invading nucleic acids from exogenous genetic elements into their CRISPR arrays and generate a memory of past invaders. When re-exposed to the specific foreign genetic element, CRISPR-cas systems target and specifically cleave homologous invasive nucleic acids in the same manner that CRISPR RNAs (crRNAs) guide Cas proteins $(20,26,27)$. Numerous pathogenic genes of enterococci originate from exogenously acquired genes and CRISPR-cas systems limit the invasion of mobile genetic elements (12). Therefore, the five isolates with an absence of CRISPR-cas determinants identified in the present study are vulnerable to invasion by foreign pathogenic genes and may contribute to the generation of significant periapical lesions.

Palmer and Gilmore (12) and Burley and Sedgley (15) have indicated that the lack of CRISPR-cas is associated with resistance to multiple antibiotics. The present study attempted to evaluate the relevance of CRISPR-cas determinants in resistance to root canal irrigants. The antibacterial activity of MTAD depends on doxycycline, a tetracycline (28). The tetracycline resistance gene belongs to a conjugative transposon and is highly transferrable in E. faecalis. In the present study, the majority of the E. faecalis exhibited resistance to MTAD, with a 1-minute survival rate of $>1 \%$. E. faecalis lacking CRISPR-cas exhibited a higher resistance to MTAD. Similarly, E. faecalis isolates that lacked CRISPR-cas exhibited a higher resistance to a synthetic cationic disinfectant, CHX, compared with E. faecalis isolates that contained CRISPR-cas. Although CHX is a disinfectant, its antibacterial mechanism acts on microbial cell membranes in a similar fashion to certain antibiotics (29). However, E. faecalis isolates were susceptible to $\mathrm{NaOCl}$ regardless of whether they contained CRISPR-cas determinants. $\mathrm{NaOCl}$ is a bacteriolytic and antibacterial agent and the antibacterial mechanism of $\mathrm{NaOCl}$ is based on its high $\mathrm{pH}$ and the irreversible oxidation of the sulfhydryl (SH) groups of essential bacterial enzymes (30). For any enzyme, this irreversible oxidation of
SH groups may occur in bacteria and is not influenced by the CRISPR-cas determinant.

E. faecalis often grow by biofilms mode on the root canal post-treatment $(31,32)$. In the present study, E. faecalis isolates with a lack of a CRISPR-cas determinant demonstrated higher biofilm formation than those containing a CRISPR-cas determinant. The CRISPR-cas determinant in bacteria defends against foreign DNA (9). E. faecalis without CRISPR-cas determinants may be invaded by mobile elements, including plasmids, prophages and pathogenicity islands (PAI) (12). The E. faecalis PAI is extensively distributed among the different strains, contains a highly variable gene, and encodes a number of pathogenicity factors, including cytolysin, aggregation substance and enterococcal surface protein, which are involved in bacterial biofilm and colonization (33). Thus, E. faecalis from a root canal infection may adhere to the bottom of the plates and generate a great deal of biofilm, possibly due to the presence of adhesive proteins. The E. faecalis PAI originates from an exogenous horizontal gene transfer and contains $\geq 100$ additional genes with unknown roles that are possibly associated with the biofilm formation capacity, virulence and drugresistance of E. faecalis (34). Therefore, the CRISPR-cas system is able to prevent mobile gene transfers and E. faecalis gains more virulence factors in the absence of a CRISPR-cas determinant.

E. faecalis, a common nosocomial pathogen, has been widely studied for antibiotic selection, gastrointestinal colonization, endocarditis and nosocomial infection (11,35-37). CRISPR-Cas is an RNA-mediated adaptive immune system that is widespread in archaea and bacteria (38). However, the role of the CRISPR-cas of bacteria in periapical periodontitis has remained elusive. The results of the aforementioned studies suggested that a CRISPR-cas determinant may regulate the biofilm formation and intracanal irrigant resistance of E. faecalis root canal isolates, and further influence the degree of periapical lesion formation. However, it must be considered that the degree of the periapical lesion depends on multiple factors and the CRISPR-cas of an E. faecalis pathogenic bacterium might only be partly responsible for the periapical lesion. E. faecalis is a predominant pathogen in post-treatment root canal infections. A further important consideration is whether CRISPR-cas regulates horizontal pathogenic gene transfer between E. faecalis and other root canal pathogens, which may determine the amount of virulence factor dissemination between the root canal pathogens and further influence the development of periapical lesions.

In conclusion, CRISPR-cas may serve a role in E. faecalis pathogenicity and periapical periodontitis. E. faecalis root canal isolates lacking CRISPR-cas determinants may exhibit greater resistance to intracanal irrigants, undergo stronger biofilm formation and generate significant periapical lesions. However, further studies on additional rot canal isolate strains of E. faecalis are required to determine the relevance of E. faecalis CRISPR-cas in drug-resistance, biofilms and periapical lesions.

\section{Acknowledgements}

The authors are grateful to Elsevier Language Editing Services for editing the draft of this manuscript. The present study was 
supported by grants from the Guangdong Natural Science Foundation (nos. S2013040014932 and 2014A030313026).

\section{References}

1. Sakamoto M, Siqueira JF Jr, Rocas IN and Benno Y: Molecular analysis of the root canal microbiota associated with endodontic treatment failures. Oral Microbiol Immunol 23: 275-281, 2008.

2. Siqueira JF Jr and Rocas IN: Diversity of endodontic microbiota revisited. J Dent Res 88: 969-981, 2009.

3. Zoletti GO, Siqueira JF Jr and Santos KR: Identification of Enterococcus faecalis in root-filled teeth with or without periradicular lesions by culture-dependent and -independent approaches. J Endod 32: 722-726, 2006.

4. Siqueira JF Jr and Rocas IN: Polymerase chain reaction-based analysis of microorganisms associated with failed endodontic treatment. Oral Surg Oral Med Oral Pathol Oral Radiol Endod 97: 85-94, 2004

5. Rocas IN, Siqueira JF Jr and Santos KR: Association of Enterococcus faecalis with different forms of periradicular diseases. J Endod 30: 315-320, 2004.

6. Stuart CH, Schwartz SA, Beeson TJ and Owatz CB Enterococcus faecalis: Its role in root canal treatment failure and current concepts in retreatment. J Endod 32: 93-98, 2006.

7. Liu H, Wei X, Ling J, Wang W and Huang X: Biofilm formation capability of Enterococcus faecalis cells in starvation phase and its susceptibility to sodium hypochlorite. J Endod 36: 630-635, 2010.

8. Figdor D, Davies JK and Sundqvist G: Starvation survival, growth and recovery of Enterococcus faecalis in human serum. Oral Microbiol Immunol 18: 234-239, 2003.

9. Barrangou R and Marraffini LA: CRISPR-Cas systems: Prokaryotes upgrade to adaptive immunity. Mol Cell 54: 234-244, 2014

10. Molloy S: Bacterial genetics: Hitting the CRISPR target. Nat Rev Microbiol 10: 376-377, 2012

11. Palmer KL, Kos VN and Gilmore MS: Horizontal gene transfer and the genomics of enterococcal antibiotic resistance. Curr Opin Microbiol 13: 632-639, 2010.

12. Palmer KL and Gilmore MS: Multidrug-resistant enterococci lack CRISPR-cas. MBio 1: e00227, 2010.

13. Palmer KL, Godfrey P, Griggs A, Kos VN, Zucker J, Desjardins C, Cerqueira G, Gevers D, Walker S, Wortman J, et al: Comparative genomics of enterococci: Variation in Enterococcus faecalis, clade structure in E. faecium and defining characteristics of E. gallinarum and E. casseliflavus. MBio 3: e00311-e00318, 2012.

14. Paulsen IT, Banerjei L, Myers GS, Nelson KE, Seshadri R, Read TD, Fouts DE, Eisen JA, Gill SR, Heidelberg JF, et al: Role of mobile DNA in the evolution of vancomycin-resistant Enterococcus faecalis. Science 299: 2071-2074, 2003.

15. Burley KM and Sedgley CM: CRISPR-Cas, a prokaryotic adaptive immune system, in endodontic, oral and multidrug-resistant hospital-acquired Enterococcus faecalis. J Endod 38: 1511-1515, 2012.

16. Zhang Q, Rho M, Tang H, Doak TG and Ye Y: CRISPR-Cas systems target a diverse collection of invasive mobile genetic elements in human microbiomes. Genome Biol 14: R40, 2013.

17. Lindenstrauss AG, Pavlovic M, Bringmann A, Behr J, Ehrmann MA and Vogel RF: Comparison of genotypic and phenotypic cluster analyses of virulence determinants and possible role of CRISPR elements towards their incidence in Enterococcus faecalis and Enterococcus faecium. Syst Appl Microbiol 34: 553-560, 2011.
18. Tong Z, Ling J, Lin Z, Li X and Mu Y: The effect of MTADN on 10 Enterococcus faecalis isolates and biofilm: an in vitro study. J Endod 39: 674-678, 2013.

19. Tong Z, Zhang Y, Ling J, Ma J, Huang L and Zhang L: An in vitro study on the effects of nisin on the antibacterial activities of 18 antibiotics against Enterococcus faecalis. PLoS One 9: e89209, 2014.

20. Makarova KS, Haft DH, Barrangou R, Brouns SJ, Charpentier E, Horvath P, Moineau S, Mojica FJ, Wolf YI, Yakunin AF, et al: Evolution and classification of the CRISPR-Cas systems. Nat Rev Microbiol 9: 467-477, 2011.

21. Andrup L: Conjugation in gram-positive bacteria and kinetics of plasmid transfer. APMIS Suppl 84: 47-55, 1998.

22. Weigel LM, Clewell DB, Gill SR, Clark NC, McDougal LK, Flannagan SE, Kolonay JF, Shetty J, Killgore GE and Tenover FC: Genetic analysis of a high-level vancomycin-resistant isolate of Staphylococcus aureus. Science 302: 1569-1571, 2003.

23. Sundqvist G, Figdor D, Persson S and Sjögren U: Microbiologic analysis of teeth with failed endodontic treatment and the outcome of conservative re-treatment. Oral Surg Oral Med Oral Pathol Oral Radiol Endod 85: 86-93, 1998.

24. Peciuliene V, Balciuniene I, Eriksen HM and Haapasalo M: Isolation of Enterococcus faecalis in previously root-filled canals in a lithuanian population. J Endod 26: 593-595, 2000.

25. Zehnder M and Guggenheim B: The mysterious appearance of enterococci in filled root canals. Int Endod J 42: 277-287, 2009.

26. Marraffini LA: CRISPR-Cas immunity against phages: Its effects on the evolution and survival of bacterial pathogens. PLoS Pathog 9: e1003765, 2013.

27. Barrangou R: CRISPR-Cas systems and RNA-guided interference. Wiley Interdiscip Rev RNA 4: 267-278, 2013.

28. Singla MG, Garg A and Gupta S: MTAD in endodontics: an update review. Oral Surg Oral Med Oral Pathol Oral Radiol Endod 112: e70-e76, 2011

29. Kanisavaran ZM: Chlorhexidine gluconate in endodontics: An update review. Int Dent J 58: 247-257, 2008.

30. Mohammadi Z: Sodium hypochlorite in endodontics: An update review. Int Dent J 58: 329-341, 2008.

31. Duggan JM and Sedgley CM: Biofilm formation of oral and endodontic Enterococcus faecalis. J Endod 33: 815-818, 2007.

32. Al-Ahmad A, Müller N, Wiedmann-Al-Ahmad M, Sava I, Hübner J, Follo M, Schirrmeister J and Hellwig E: Endodontic and salivary isolates of Enterococcus faecalis integrate into biofilm from human salivary bacteria cultivated in vitro. J Endod 35: 986-991, 2009.

33. Shankar N, Baghdayan AS and Gilmore MS: Modulation of virulence within a pathogenicity island in vancomycin-resistant Enterococcus faecalis. Nature 417: 746-750, 2002.

34. Manson JM, Hancock LE and Gilmore MS: Mechanism of chromosomal transfer of Enterococcus faecalis pathogenicity island, capsule, antimicrobial resistance and other traits. Proc Natl Acad Sci USA 107: 12269-12274, 2010.

35. Hollenbeck BL and Rice LB: Intrinsic and acquired resistance mechanisms in enterococcus. Virulence 3: 421-433, 2012.

36. Franz CM, Huch M, Abriouel H, Holzapfel W and Gálvez A: Enterococci as probiotics and their implications in food safety. Int J Food Microbiol 151: 125-140, 2011.

37. Dahl A and Bruun NE: Enterococcus faecalis infective endocarditis: Focus on clinical aspects. Expert Rev Cardiovasc Ther 11: 1247-1257, 2013.

38. Wiedenheft B, Sternberg SH and Doudna JA: RNA-guided genetic silencing systems in bacteria and archaea. Nature 482: 331-338, 2012. 